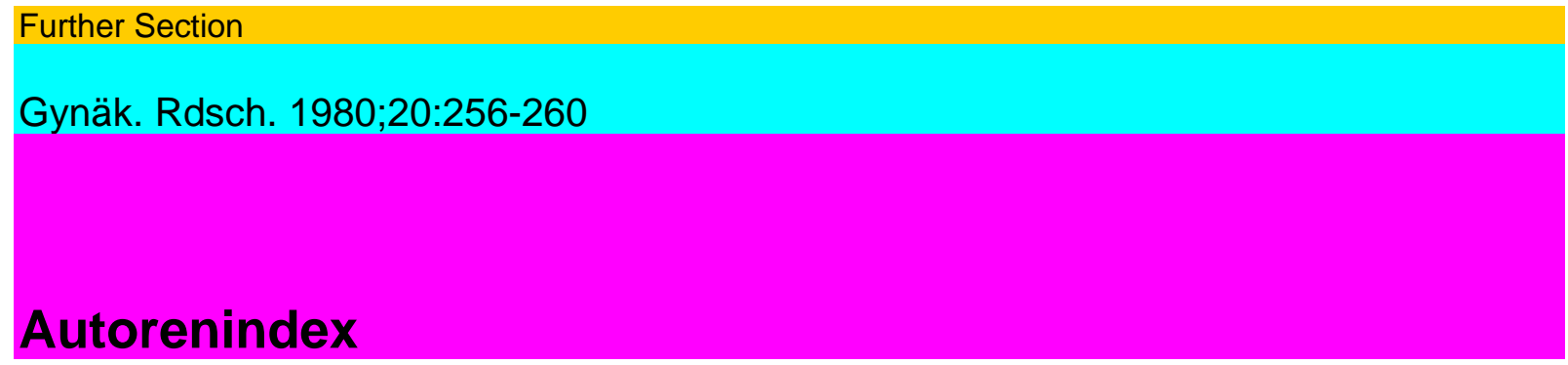

Aksu, M.F. 251 Alder, W. 56 Anderson, I. 38 Andersson, K.E. 235 Andor, J. 80 (Suppl. 1)

Antoniadis, R. 132 (Suppl. 1)

Bänninger, U. 126 (Suppl. 1)

Baltisser, I. 134 (Suppl. 1)

Baltzer, J. 191

Barthel, R. 133

Bartl, W. 148 (Suppl. 2)

Battista, H.J. 268 (Suppl. 2)

Beck, L. 1 (Suppl. 1)

Becker, H. 241,254 (Suppl. 2)

Beckmann, M. 59

Béguin, F. 103 (Suppl. 1)

Benz, J. 45 (Suppl. 1), 74 (Suppl. 1),

86 (Suppl. 1), 92 (Suppl. 1) Berger, C. 52 (Suppl. 1) Bergink,E.W. 247

Bernaschek, G. 18 (Suppl. 2), 208 (Suppl. Bichler, A. 239 (Suppl. 2) Bieglmayer, C. 179

Binswanger, U. 1 Bischoff, J. 241 Bonilla-Musoles, F. 218 Borell,J. 243

Bossart, H. 65 (Suppl. 1),67 (Suppl. 1) Bowers, E.J. 44 Brabec.W. 230 (Suppl. 2) Breitenecker, G. 109 (Suppl. 2), 148

(Suppl. 2), 217 (Suppl. 2) Brehm,R. 281 (Suppl. 2) Brehme,K. 121 Breitfellner, G. 279 (Suppl.

2) Bretscher, J. 87 (Suppl. 1), 90 (Suppl. 1),

109 (Suppl. 1) Briel, R.C. 237 Briner, U. 29

Brunhöber, J. 50 Büchi,K. 83 (Suppl. 1) Bürgi, W. 94 (Suppl. 1) Burghardt, E. 103 (Suppl. 2)

Campana, A. 141 (Suppl. 1) Cimber, H. 130 (Suppl. 1) Clelland, R.C. 44 Clinch, J. 39 Colle,E. 183 Crevoisier, Ch.-A. 171

Dadak,C. 18 (Suppl. 2)

Dame,W.R. 59

Dapunt, O. 67 (Suppl. 2), 173 (Suppl. 2),

230 (Suppl. 2) Dbaly, J. 130 (Suppl. 1) Deckart, H. 50 Denk, H. 148 (Suppl. 2) Desgranges, M.F. 183 Dienstl, F. 268 (Suppl. 2) Diserens, B. 128 (Suppl. 1) Dobrovits, H. 131 (Suppl. 2) Döring, G.K. 57 Drähne,A. 237 Dreher,E. 113 (Suppl. 1)

Eberhard, J. 49 (Suppl. 1), 124 (Suppl. 1),

134 (Suppl. 1) Ebert, C. 59 Eckert, M. 171 Einhorn,N. 121 Eliot, E.W. 180

Elsner-Mackay, P. 235 (Suppl. 2) Endler, M. 212 (Suppl. 2) Euller. A. 58 (Suppl. 2), 72 (Suppl.

2)

Feichtinger, W. 58 (Suppl. 2) Fehlauer, K.U. 130 Fenske, M. 239

Autorenindex

257

Fischer, M. 77 (Suppl. 1) 
Fischl,F. 200

Floersheim, Y. 81 (Suppl. 1), 83 (Suppl. 1)

Frangenheim, H. 142 (Suppl. 1)

Frei, D. 1

Freilinger, G. 46 (Suppl. 2)

Fresenius, K.J. 57

Fricker, H.S. 106 (Suppl. 1)

Friedrich, F. 217 (Suppl. 2)

Frimmel, H. 148 (Suppl. 2)

Frischknecht, M. 49 (Suppl. 1)

Gall, S.A. 128

Gamulin, Z. 14 (Suppl. 1)

Gaudenz, R. 89, 123 (Suppl. 1)

Gavazzi, G. 243

Gelfand,M.M. 183

Gemperle,M. 14 (Suppl. 1)

Gerber. C. 72 (Suppl. 1)

Gerstner, G. 64 (Suppl. 2), 274 (Suppl. 2),

277 (Suppl. 2) Gigon,U. 63 (Suppl. 1), 141 (Suppl. 1) Girndt, J. 239 Gitsch,E. 3 (Suppl. 2), 118 (Suppl. 2),

195 (Suppl. 2), 247 (Suppl. 2) Golob,E. 261 (Suppl. 2), 285 (Suppl. 2) Greenblatt, R.B. 251

Grosch, G. 50 Grospietsch, G. 239 Gruber, U.F. 125 Grünberger, V. 131 (Suppl. 2) Grünberger, W. 203 (Suppl. 2), 205

(Suppl. 2) Guülermin, R. 103 (Suppl. 1)

Haensel,W. 133 Halberstadt, E. 56 (Suppl. 1) Haldemann, R. 171 Haller,H. 261 (Suppl. 2)

Hammond, Ch.B. 189 Hardmeier, T. 134 (Suppl. 1) Harlap,S. 129 Haspels, A.A. 253 Hauser,

G.A. 117 (Suppl. 1) Hauser, H. 138 (Suppl. 1)

Heinzl, S. 69 (Suppl. 1), 136 (Suppl. 1)

Heizmann, P. 171

Herbst, S. 48 (Suppl. 1)

Herson, J. 46

Herzog, B. 185

Hetzel, H. 151 (Suppl. 2), 173 (Suppl. 2)

Hickey, R.J. 44

Hodge, M.P. 46

Hoffelner, L. 131 (Suppl. 2)

Hofmann, H. 258 (Suppl. 2)

Holm, P. 130

Holzer, E. 113 (Suppl. 2), 117

Holzner, J.H. 11 (Suppl. 2)

Horstschäfer, W. 51 (Suppl. 1)

Howat, R.C.L. 180

Huber, A. 288 (Suppl. 2)

Huber, J. 274 (Suppl. 2)

Hussleín,P. 179

Ingold, W. 45 (Suppl. 1), 74 (Suppl. 1) 
Jänne, O. 177

Jalùvka, V. 11

Janecek, P. 65 (Suppl. 1), 67 (Suppl. 1)

Janisch, H. 98 (Suppl. 2), 142 (Suppl. 2),

148 (Suppl. 2), 277 (Suppl. 2) Jerabek, R. 67 (Suppl. 2) Johnson, T.R., Jr. 41 Jorde, A. 50 Juchli, P. 134 (Suppl. 1)

Käch, O. 62 (Suppl. 1)

Kaufman, D.W. 176

Kauppila, A. 177

Keller, P.J. 72 (Suppl. 1), 74 (Suppl. 1),

77 (Suppl. 1), 81 (Suppl. 1), 83 (Suppl. 1) Kemeter, P. 58 (Suppl. 2), 217 (Suppl. 2) Kern, F. 19

(Suppl. 1) Kiesler, J. 279 (Suppl. 2) Kissel, U. 48 Klopper, A. 249 Klug, P.W. 177 (Suppl. 2)

Knitza, E. 53 (Suppl. 1) Knitza, R. 53 (Suppl. 1)

Autorenindex

258

Koepcke,W. 191

Kofler, E. 274 (Suppl. 2), 277 (Suppl. 2)

Kogelnik, H.D. 135 (Suppl. 2)

Kontula, K. 177

Kratochwil, A. 18 (Suppl. 2), 208 (Suppl. 2)

Kreel, B.K. van 236

Kreienberg, R. 193

Kreienbühl, G. 49 (Suppl. 1)

Kruse,H.J. 130

Kubista, E. 33 (Suppl. 2), 35 (Suppl. 2),

110 (Suppl. 2) Kucera, H. 5 (Suppl. 2), 110 Kuchen, J.D. 105 (Suppl. 1) Kuhn,W. 239 Künsch, U. 108 (Suppl. 1) Kuntner, L. 162 Kunz, J. 126 (Suppl. 1) Kunz,S. 237 Kupka, S. 118 (Suppl. 2), 191

(Suppl. 2)

Lahodny,J. 186 (Suppl. 2)

Lahousen, M. 77 (Suppl. 2), 113 (Suppl. 2)

Landolt, A. 45 (Suppl. 1), 74 (Suppl. 1)

Lange, E. 271 (Suppl. 2)

Lanz, R. 132 (Suppl. 1)

Lauritzen, C. 124

Lechner, W. 268 (Suppl. 2)

Lemarchand, M.-T. 135

Lemmel,E.-M. 193

Lemon, H.M. 248

Leodolter, S. 127 (Suppl. 2), 191 (Suppl. 2)

Lepuschütø, H. 84 (Suppl. 2)

Lichtenegger, W. 254 (Suppl. 2)

Lienhard, P. 124 (Suppl. 1)

Limota, P. 243

Lindgren, J. 54 


\section{Ling,W.Y. 245}

Lippert, T.H. 131

Litschgi, M. 52 (Suppl. 1), 92 (Suppl. 1),

141 (Suppl. 1) Ludescher, E. 264 (Suppl. 2) Ludwig, H. 138 (Suppl. 2) Lüscher, K.P. 62 (Suppl. 1), 76 (Suppl. 1),

108 (Suppl. 1)

Mayer, H.G.K. 177 (Suppl. 2)

Mayer, H.O. 220 (Suppl. 2)

Mack, A.E. 180

Mac Leod, S. 245

Madersbacher, H. 161 (Suppl. 2)

Maleika, F. 141 (Suppl. 1)

Manzl, J. 151 (Suppl. 2), 173 (Suppl. 2),

227 (Suppl. 2) Maroni, E. 101 (Suppl. 1) Marti, A. 76 (Suppl. 1) Martini, L. 243 Masson,M. 183

Mattingly, R.F. 43 McEwan, J. 126 Menon, J. 41 Menon, K.M.J. 41 Menon, M. 41 Meissner, M. 187 Melchert, F. 193 Meyer-Breiting, P. 56 (Suppl. 1) Miettinen, O.S. 176 Miller, J.M., Jr. 189 Mönkemeier, D. 85 Morf, P. 96 (Suppl. 1), 207 Mühlenstedt, D. 52, 187 Müller, H.R. 94 (Suppl. 1)

Nagl, F. 123 (Suppl. 2) Nasr, F. 58 (Suppl. 2) Neuenschwander, F. 29, 99 (Suppl. 1) Neumann, H. 235 (Suppl. 2) Nigg, O. 138 (Suppl. 1) Nowara, P. 48

Nürnberger, N. 181 (Suppl. 2), 191 (Suppl. 2)

Obel, E. 182

Oberholzer, F. 49 (Suppl. 1)

Odermatt, H. 101 (Suppl. 1)

Ortner, A. 67 (Suppl. 2), 227 (Suppl. 2)

Papageorgiou, A.N. 183 Pattillo, R.A. 43 Pavelka, R. 223 (Suppl. 2) Pellicier, A. 218

Autorenindex

259

Perez-Gil, M. 218

Pfeiffer, K.M. 132 (Suppl. 1)

Pfleiderer, A. 48

Pflüger, H. 191 (Suppl. 2)

Philipp, K. 127 (Suppl. 2), 198, 281

(Suppl. 2) Pickel, H. 77 (Suppl. 2), 84 (Suppl. 2),

90 (Suppl. 2) Pipard,G. 138 (Suppl. 1) Piva, F. 243

Popp, L.W. 100 (Suppl. 1) Prestele,H. 241 Pürstner, P. 90 (Suppl. 2), 258

(Suppl. 2)

Quakernack, K. 59 Quigley, M.E. 42

Radakovic, D. 27 (Suppl. 1), 69 (Suppl. 1)

Rais, M. 135

Ramedi de Gomez, G. 22

Ramzin, M.S. 48 (Suppl. 1)

Rauch, R. 86 (Suppl. 1), 92 (Suppl. 1)

Rebhan,E.-M. 241

Reddy, S. 38 
Reinartz, G. 135 (Suppl. 2)

Reinold, E. 223 (Suppl. 2)

Rey-Stocker, I. 135

Richie, R. 171

Richter, D. 48

Richter, R. 112 (Suppl. 1)

Riedmann, R. 77 (Suppl. 1)

Riss, P. 212 (Suppl. 2), 223 (Suppl. 2)

Robichaud, A. 245

Rodriquez, J. 41

Rosanelli, K. 246 (Suppl. 2)

Rosegger, H. 246 (Suppl. 2)

Rosenberg, L. 176

Rottenberg, R. 138 (Suppl. 1)

Rotter, M. 274 (Suppl. 2)

Ruckert, A.C.F. 43

Rückert, E. 50

Rutledge, F. 46

Ruttensteiner, J. 285 (Suppl. 2)

Ryser,W. 130 (Suppl. 1)

Sager. W.D. 84 (Suppl. 2),

254 (Suppl. 2) Salmi. T. 122,249 Salzer, H. 127 (Suppl. 2), 144 (Suppl. 2),

195 (Suppl. 2), 199 (Suppl. 2), 217

(Suppl. 2) Sauter, M. 106 (Suppl. 1) Schäfer, P. 54 (Suppl. 1) Schaer, H.M. 59 (Suppl. 1) Scharf, O. 67 (Suppl. 2) Schellenberg, J.-C. 8 (Suppl. 1) Schmid, R. 39 (Suppl. 2), 201

(Suppl. 2) Schmidt-Gollwitzer, K. 102 Schmidt-Goll·witzer, M. 102 Schneider, H.P.G. 52, 187 Schneider, P. 80 (Suppl. 1) Schrödl, P. 90 (Suppl. 2) Schüssler, B. 102 Schurz, A.R. 264 (Suppl. 2), 266 (Suppl. 2),

279 (Suppl. 2) Seitz,W. 135 (Suppl. 2) Sen, K.K. 41 Seppälä, M. 54 Seski, J.C. 41 Shapiro, S. 176 Shatz,R. 183 Sheehan.K.L. 42 Shekleton,P. 38 Slone, D 176 Smith, J.P. 46 Sormani,H. 48 (Suppl. 1) Spelina, P. 113 (Suppl. 1) Spernol, R. 212 (Suppl. 2) Spinelli, A. 108 (Suppl. 1) Spitzy,K.H. 196 Spona, J. 179 Stamm, H.-E. 65 Steiner. I. 144 (Suppl. 2) Steuble,E. 124 (Suppl. 1) Stirmer, V. 69 (Suppl. 1) Stockhammer, P. 133 Stoffel, R. 87 (Suppl. 1), 90 (Suppl. 1), 109 (Suppl. 1) Stoll, U. 65 (Suppl. 1)

Autorenindex

260

Stoll, W. 90 (Suppl. 1), 94 (Suppl. 1),

106 (Suppl. 1) Stolley, P.D. 176 Story, M.T. 43 Stühmer, M. 185 Stünzner, D. 220 (Suppl. 2) Stulz,P. 132 (Suppl. 1) Stummvoll,W. 123 (Suppl. 2) Suppan, P. 65 (Suppl. 1), 67 (Suppl. 1)

Surwit, E.A. 189 Syrjälä, P. 177 Szalay, S. 98 (Suppl. 2), 142 (Suppl. 2), 144 (Suppl. 2), 148 (Suppl. 2), 203 Szalmay,G. 141 (Suppl. 1) Szepesi, T. 135 (Suppl. 2)

Tatra, G. 138 (Suppl. 2), 142 (Suppl. 2) Tapia, J.E. 117 (Suppl. 1) Tauber,P.F. 141 (Suppl. 1) Thiessen, P. 48 Tempfer, H. 72 (Suppl. 2) Tibi, B. 48 Tölle,E. 242 Topolanski-Sierra, R. 22 Tscherne, K. 80 (Suppl. 1) Tsingounis, V.A. 251 Tulzer,H. 203 (Suppl. 2) Turina, D. 81 (Suppl. 1) 
Uehlinger, J. 86 (Suppl. 1) Ulm, R. 179 Ulmsten,U. 235 Urdl, W. 90 (Suppl. 2) Utian, W.H. 252 Vetter,K. 45 (Suppl. 1), 72 (Suppl. 1)

Vihko,R. 177

Vögelin, E. 80 (Suppl. 1)

Volkmar, H. 130

Vuletic,N. 69 (Suppl. 1)

Wagner, G. 64 (Suppl. 2), 191 (Suppl. 2), 195 (Suppl. 2), 199 (Suppl. 2)

Wahlström,T. 54

Wallenburg, H.C.S. 236

Washüttl, J. 144 (Suppl. 2)

Weber, T. 182

Weil, A. 89, 123 (Suppl. 1)

Wharton, J.T. 46

Weiss, P.A.M. 242 (Suppl. 2)

Wickings, E.J. 52

Wilkes, M.M. 42

Wingerup, L. 235

Winter, R. 242 (Suppl. 2), 250 (Suppl. 2), 258 (Suppl. 2)

Wintsch,K. 106 (Suppl. 1)

Wirth, T. 86 (Suppl. 1), 92 (Suppl. 1)

Witschi, M. 99 (Suppl. 1)

Wix,P. 52

Wolf, G. 26 (Suppl. 2)

Wood, C. 38

Wrixon,W. 245

Wyss,H. 80 (Suppl. 1)

Yen, S.S.C. 42

Zach,H.P. 185 Zahn, V. 185 Zayid,I. 245 Zufferey, M.-M. 135 Zwicker, H. 56 